\title{
BMJ Open Increasing engagement in advance care planning using a behaviour change model: study protocol for the STAMP randomised controlled trials
}

\author{
Terri R Fried, ${ }^{1,2}$ Colleen A Redding, ${ }^{3,4}$ Steven Martino, ${ }^{5,6}$ Andrea Paiva, ${ }^{3,4}$ \\ Lynne lannone, ${ }^{2,7}$ Maria Zenoni, ${ }^{2,7}$ Laura A Blakley, ${ }^{5,6}$ Joseph S Rossi, ${ }^{3,4}$ \\ John O'Leary ${ }^{2,7}$
}

To cite: Fried TR, Redding CA, Martino S, et al. Increasing engagement in advance care planning using a behaviour change model: study protocol for the STAMP randomised controlled trials. BMJ Open 2018;8:e25340. doi:10.1136/ bmjopen-2018-025340

- Prepublication history for this paper is available online. To view these files, please visit the journal online (http://dx.doi org/10.1136/bmjopen-2018025340).

Received 10 July 2018 Revised 13 July 2018 Accepted 16 July 2018

Check for updates

(c) Author(s) (or their employer(s)) 2018. Re-use permitted under CC BY-NC. No commercial re-use. See rights and permissions. Published by BMJ.

For numbered affiliations see end of article.

Correspondence to

Dr Terri R Fried;

terri.fried@yale.edu

\section{ABSTRACT}

Introduction Advance care planning (ACP) is a key component of high-quality end-of-life care but is underused. Interventions based on models of behaviour change may fill an important gap in available programmes to increase ACP engagement. Such interventions are designed for broad outreach and flexibility in delivery. The purpose of the Sharing and Talking about My Preferences study is to examine the efficacy of three behaviour change approaches to increasing ACP engagement through two related randomised controlled trials being conducted in different settings (Veterans Affairs (VA) medical centre and community).

Methods and analysis Eligible participants are 55 years or older. Participants in the community are being recruited in person in primary care and specialty outpatient practices and senior living sites, and participants in the VA are recruited by telephone. In the community, randomisation is at the level of the practice or site, with all persons at a given practice/ site receiving either computer-tailored feedback with a behaviour stage-matched brochure (computer-tailored intervention (CTI)) or usual care. At the VA, randomisation is at the level of the participant and is stratified by the number of ACP behaviours completed at baseline. Participants are randomised to one of four groups: CTI, motivational interviewing, motivational enhancement therapy or usual care. The primary outcome is completion of four key ACP behaviours: identification of a surrogate decision maker, communication about goals, completing advance directives and ensuring documents are in the medical record. Analysis will be conducted using mixed effects models, taking into account the clustered randomisation for the community study. Ethics and randomisation The studies have been approved by the appropriate Institutional Review Boards and are being overseen by a Safety Monitoring Committee. The results of these studies will be disseminated to academic audiences and leadership in in the community and VA sites. Trial registration numbers NCT03137459 and NCT03103828.

\section{INTRODUCTION}

The Institute of Medicine (IOM) report, 'Dying in America: Improving Quality and Honoring Individual Preferences near the
Strengths and limitations of this study

- The intervention is based on a comprehensive model of advance care planning (ACP) that focuses on enhancing engagement and improving communication among patients, their surrogates and their providers.

- The behavioural health approach to ACP provides a framework for practical interventions that can be implemented in a wide variety of settings.

- The interventions have been developed in English only, and the study therefore excludes individuals whose primary language is other than English.

- The study is being conducted in a single geographic region and therefore may have limited generalisability.

End of Life' devotes an entire chapter to advance care planning (ACP), the process by which patients can plan for the care they will receive if they become incapable of participating in medical decision making. ${ }^{1}$ The report endorses the promise of ACP to provide 'a measure of control over the final phase of life' and to ensure that 'patients' wishes are known and respected to the extent possible'. ACP is also associated with improved caregiver outcomes. ${ }^{2}{ }^{3}$ However, as outlined in the IOM report chapter, ACP remains underused. A recent systematic review concluded that only approximately one-third of adults in the USA has completed advance directives (ADs). ${ }^{4}$ Moreover, ADs alone are not sufficient in the absence of efforts to promote communication. ${ }^{5}$

Several intervention approaches have demonstrated efficacy in increasing engagement in ACP. Respecting Choices, consisting of facilitated discussions lasting between 60 min and $90 \mathrm{~min}$ between patients and their surrogates, increased surrogates' knowledge of patients' preferences and reduced 
caregiver stress. ${ }^{67}$ The PREPARE website, a self-administered tool providing step-by-step processes for ACP supplemented with video stories and modelling of behaviours, increased ACP documentation. ${ }^{8}$ Additional approaches may be necessary to bridge the gap between intensive clinician-led and self-administered tools for ACP engagement. For example, efforts to replicate and disseminate the Respecting Choices programme in a large metropolitan area required 'concerted and sustained leadership', a prolonged planning phase and the subsidising of salaries. ${ }^{9}$ This experience suggests that intensive programmes are best targeted to selected patients at high risk of facing difficult treatment choices. The IOM report supports such targeting, suggesting a lifespan approach to ACP, beginning early with broad considerations of wishes that become more clinical and detailed over time. This lifespan strategy is congruent with our approach, which is designed to promote widespread dissemination of material that engages individuals in midlife and works to give them the tools to help them reconsider their wishes over time as health challenges become clearer. While the PREPARE website provides a thorough introduction to ACP, over $50 \%$ of participants had no access to the internet, and the website was viewed in research offices, ${ }^{8}$ suggesting that web-based materials may have limited outreach.

The Sharing and Talking about My Preferences (STAMP) study was designed to address gaps in the existing programmes for promoting participation in ACP. The STAMP interventions are based on the conceptual model of ACP considered as a set of inter-related health behaviours. ${ }^{10}$ Prior research has demonstrated that participants have variable readiness to engage in ACP behaviours ${ }^{10}$ and that this readiness can be represented and explained by constructs of the Transtheoretical Model (TTM), including stages of change, decisional balance (the pros and cons of behaviour change) and processes of change. ${ }^{11}$ Readiness is also explained by a construct of values/beliefs. Unlike the pros and cons, which are factually verifiable, values and beliefs consist of common misperceptions about ACP and religious values that can function as barriers to ACP. ${ }^{11}$

STAMP evaluates three behaviour change approaches to promoting ACP engagement. The first is the use of individualised feedback reports with stage-matched brochures. ${ }^{12}$ The second is telephone-delivered motivational interviewing (MI), and the third is telephone-delivered motivational enhancement therapy (MET), a combination of written feedback and MI. The health behaviours consist of: (A) identifying a trusted individual to act as a surrogate decision maker or healthcare agent; (B) communicating with this person about goals, preferences and values; (C) completing $\mathrm{ADs}$ (formal assignment of healthcare agent and living will); and (D) ensuring both that the physician is aware of documents and that documents are in the medical record. STAMP consists of two related randomised controlled trials (RCTs). The hypothesis is that individuals receiving each of the behaviour change approaches will be more likely than individuals receiving usual care to complete all of the health behaviours.

\section{METHODS AND ANALYSIS \\ Study overview}

STAMP consists of two related RCTs, with shared inclusion and exclusion criteria for study participation, measures, outcomes and analytic approaches but with enrolment procedures and interventions adapted for each of the two trials. Each RCT will test the efficacy of one or more interventions on the proportion of study participants who complete participation in ACP compared with usual care. One RCT is being conducted within the primary care clinics of the Veterans Affairs Connecticut Healthcare System (subsequently referred to as the VA study), and the second is in community-based primary care practices and senior living communities in the greater New Haven area (subsequently referred to as the community study). Trial registration data are provided in table 1 .

\section{Participants, recruitment and enrolment}

Inclusion criteria include: age 55 years or older and, for participants recruited in the VA study, having a primary care clinic visit within the last 12 months. Exclusion criteria include: severe hearing or vision loss, moderate-to-severe cognitive impairment identified by chart review (VA study only), physician diagnosis, Brief Orientation Memory Concentration test score $>10^{13}$ or inability to participate in the process of informed consent; primary language other than English; active psychiatric illness (current symptoms of depression, anxiety, substance abuse or psychosis), no regular access to a telephone; no permanent mailing address; completion of all four ACP behaviours; or lack of physician permission for participation.

Screening, recruitment and enrolment for participants differs according to study. In the VA study, potential participants are selected from a list obtained under a Health Insurance Portability and Accountability Act waiver of all persons age 55 years and older who have had a primary care visit within the last year at VA Connecticut. This method of identifying participants accomplishes two objectives. First, it facilitates oversampling of women and minorities to ensure adequate representation in the study population. The study is aiming for $25 \%$ women and $40 \%$ non-white participants. Second, it allows for all study procedures to be done by telephone. Veterans relying on public transportation face a significant barrier to access, with only $25 \%$ living within a $60 \mathrm{~min}$ transit time from a VA medical facility. ${ }^{14}$ The ability to identify individuals and deliver the intervention by telephone is in keeping with the VA's investment in telehealth to improve access to a variety of services. ${ }^{15}$ Chart screens are performed to identify exclusion criteria. Potentially eligible participants are sent an opt-out letter. If they do not opt out, a research assistant calls the participant to explain the study and completes the screening for eligibility. If the 
Table 1 Trial registration data

\begin{tabular}{|c|c|c|}
\hline Category & STAMP (Community) & STAMP (VA) \\
\hline $\begin{array}{l}\text { Primary registry and trial identifying } \\
\text { number }\end{array}$ & $\begin{array}{l}\text { ClinicalTrials.gov } \\
\text { NCT03103828 }\end{array}$ & $\begin{array}{l}\text { ClinicalTrials.gov } \\
\text { NCT03137459 }\end{array}$ \\
\hline $\begin{array}{l}\text { Date of registration in primary } \\
\text { registry }\end{array}$ & 27 April 2017 & 31 March 2017 \\
\hline $\begin{array}{l}\text { Source(s) of monetary or material } \\
\text { support }\end{array}$ & $\begin{array}{l}\mathrm{NIH} / \mathrm{NINR} \\
\mathrm{NIH} / \mathrm{NIA}\end{array}$ & VA HSR\&D \\
\hline Primary sponsor & $\mathrm{NIH} / \mathrm{NINR}$ & VA HSR\&D \\
\hline Secondary sponsor & None & None \\
\hline Contact for public queries & Lynne lannone, MS: Iynne.iannone@yale.edu & \\
\hline Contact for scientific queries & Terri Fried, MD: terri.fried@yale.edu & \\
\hline Public title & STAMP: Sharing and Talking about My Prefere & nces \\
\hline Scientific title & STAMP: Sharing and Talking about My Prefere & nces \\
\hline Countries of recruitment & USA & USA \\
\hline $\begin{array}{l}\text { Health condition(s) or problem(s) } \\
\text { studied }\end{array}$ & Advance care planning (ACP) & \\
\hline Intervention(s) & $\begin{array}{l}\text { Active comparator: TTM-based CTI; no } \\
\text { intervention: usual care. }\end{array}$ & $\begin{array}{l}\text { Active comparators: TTM-based CTI, MI, } \\
\text { MET; no intervention: usual care. }\end{array}$ \\
\hline Key inclusion and exclusion criteria & $\begin{array}{l}\text { Inclusion: age } 55 \text { years and older and belongir } \\
\text { community. Exclusion: severe hearing impairm } \\
\text { cognitive impairment, primary language other } \\
\text { completion of all four key ACP behaviours. }\end{array}$ & $\begin{array}{l}\text { ig to healthcare system or residential } \\
\text { ent, severe visual, moderate to severe } \\
\text { than English, active psychiatric illness, }\end{array}$ \\
\hline Study type & $\begin{array}{l}\text { Interventional; allocation: randomised; interver } \\
\text { single masking (outcomes assessor); primary }\end{array}$ & $\begin{array}{l}\text { tion model: parallel assignment; masking: } \\
\text { ourpose: health services research. }\end{array}$ \\
\hline Date of first enrolment & July 2017 & October 2017 \\
\hline Target sample size & 1000 & 484 \\
\hline Recruitment status & Recruiting & \\
\hline Primary outcome(s) & Completion of four key ACP behaviours & \\
\hline Key secondary outcomes & Stage of change for each of the four key ACP & behaviours \\
\hline
\end{tabular}

CTI, computer tailored intervention; HSR\&D, Health Services Research and Delivery; MET, motivational enhancement therapy; MI, motivational interviewing; NIA, National Institute on Aging; NIH, National Institutes of Health; NINR, National Institute of Nursing Research; TTM,

Transtheoretical Model; VA, Veterans Affairs.

participant is eligible and interested in participation, a process of verbal assent is obtained. Participants who are randomised to MI or MET are asked if they have a surrogate they would like to include in their MI/MET session. If they do, they are asked to provide the contact information for the surrogate, who must then provide verbal assent prior to inclusion in the session. All identifiable data are stored separately from study records, which are identified by code number, in access-restricted database files behind institutional firewalls. Printed records are kept in locked offices.

Recruitment in the community study is designed to encourage ACP as a part of routine healthcare. In community practices, the list of patients scheduled for either well patient or routine follow-up visits is reviewed prior to each clinic session to identify potentially eligible participants. These individuals are given an information sheet to read prior to the encounter. The clinician confirms patient interest in the study, and willing individuals meet with a research assistant at the end of the encounter to complete a process of written informed consent. In senior living communities, recruitment takes place both in the on-site medical clinics as described above and among the community as a whole. For the latter, the study is introduced in a talk given by the principal investigator, followed by the opportunity for volunteers to sign up for times to meet with the research assistant to complete written informed consent.

\section{Intervention and control conditions}

Similar to recruitment procedures, the intervention arms are tailored to take advantage of the opportunities offered by the different study settings. The availability of health psychologists and MI training at the VA supported the strategy of developing MI interventions to promote ACP engagement in addition to a computer-tailored intervention (CTI) that generates printed feedback. Therefore, in the VA study, there are four arms: CTI, MI, MET and control. In the community, there are two arms: CTI and control. All arms consist of contact at baseline, 2 months 
and 4 months with delivery of the intervention according to assignment. There is a contact at 6 months for final assessment.

\section{Computer-tailored intervention}

The development of the CTI has been previously described. ${ }^{12}$ Briefly, it is an expert system (a software system consisting of an assessment battery, normative data to make comparisons, decision rules for delivering feedback and feedback components) based on the TTM. TTM intervention principles include respecting and reflecting individuals' stages of change and their progress over time on each construct in individualised feedback reports. Such reports have been found to be effective across a wide range of other health behaviours. ${ }^{16}$ The system assesses key constructs of the TTM, including stage of change (readiness to participate in each of the ACP behaviours), decisional balance, values/beliefs and processes of change. The original system consisted of feedback paragraphs developed for each stage of the four ACP behaviours and for decisional balance, which are pulled into a templated cross-sectional, or 'normative', report. For this study, the system was further developed in order to give feedback based on simultaneous consideration of stage for all four behaviours (referred to as stage pattern) as well as to give longitudinal feedback by comparing stage at follow-up to stage at baseline ('ipsative' reporting). Each feedback report consists of: (1) introduction to ACP (common across reports); (2) figure illustrating stage of change for each behaviour (normative) and changes in stage at follow-up (ipsative) (see figure 1); (3) brief stage pattern-tailored feedback; (4) feedback for up to three endorsed values/beliefs items; (5) decisional balance by stage pattern feedback; (6) processes/strategies/efficacy or 'next steps' stage pattern-tailored feedback; and (7)

\section{How Ready Are You?}

This figure shows you how ready you are to do each of these steps towards your Advance Care Plan.

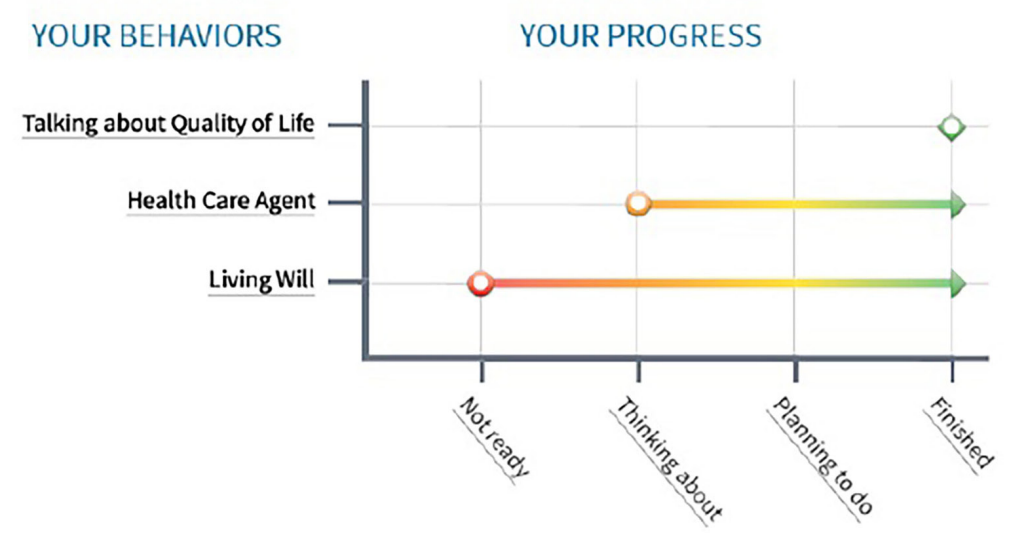

\begin{tabular}{|c|c|c|}
\hline Your forms are: & at MD Office & in Medical Record \\
\hline Healthcare Agent & $\checkmark$ & \\
\hline Living Will & $\checkmark$ \\
\hline
\end{tabular}

You told us that you are:

- Just like last time, you have finished talking with your loved ones about quality versus quantity of life.

- Last time you were thinking about naming your healthcare agent, and now you have done this. That's great!

- Last time you were not ready to complete your living will, and now you have done this. Good step forward!

Figure 1 Example of figure included in follow-up feedback report illustrating change in readiness over time for the ACP behaviours. ACP, advance care planning. 
summary. For participants in the earliest stages of change for multiple behaviours, the report provides brief 'next steps' focused on activities to help promote favourable attitudes towards ACP. For participants in later stages, the report provides suggestions for how the participant can go about engaging in the behaviour. The 'next steps' section also informs participants how completing one ACP behaviour can help in the completion of others.

The feedback reports make reference to one of two stage-matched brochures. Each of these brochures provides additional details of ACP. The first brochure, provided to participants who have not yet completed any of the ACP behaviours, provides additional information promoting the reasons for engaging in ACP and addressing potential barriers. It also contains two stories describing families who did and did not engage in ACP. The second brochure, provided to participants who have completed at least one ACP behaviour, also contains additional information about how to engage in each. In addition, this brochure contains either the VA or state of Connecticut Advance Directives Form. Finally, participants receive a fourfold pamphlet designed to be given to potential surrogate decision makers in order to explain their role in ACP.

For participants enrolled in the VA, the assessment occurs by telephone, and the feedback report, brochure, and pamphlet are mailed. For participants enrolled in the community, the initial assessment is done in person in the clinician office or private space in the senior living community, and the report is printed using a portable printer. If the participant does not have sufficient time after his or her appointment but has provided informed consent, the assessment is done by telephone at a later time. All follow-up assessments are done by telephone, with reports mailed to participants.

\section{Motivational interviewing}

The development of the MI protocol has been previously described. ${ }^{17}$ MI sessions are conducted by health psychologists and social workers who have received training by Dr Martino, a member of the research team and of the Motivational Interviewing Network of Trainers, who is an expert in training clinicians in the conduct of MI for clinical trials. ${ }^{18-20}$ The training consisted of a 2-day experiential workshop and STAMP MI/MET manual review, followed by supervised practice cases, incorporating fidelity rating-based feedback and coaching based on the review of audio recorded telephone sessions using the Independent Tape Rater Scale. ${ }^{21}$ Dr Martino reviewed and rated all practice cases for initial certification. Initial certification was defined as at least adequate or average adherence and competence ratings on three consecutive practice sessions. If a clinician's performance later drifts below the initial certification level during the trial, then Dr Martino will provide additional supervision and training and assign another practice case if necessary. The MI intervention consists of four steps: (1) elicitation and clarification of patient's understanding of and current engagement in ACP; (2) building motivation for ACP; (3) developing a change plan; and (4) summarising the overall discussion. The first step involves understanding in what ways the patient thinks ACP is important and the patient's knowledge of the four key ACP behaviours. Consistent with MI, the Elicit-Provide-Elicit approach is used to deliver ACP. This approach involves inviting the patient to share his or her knowledge, asking the patient permission to provide additional information, and then eliciting the patient's reaction to this new information. The second step consists of four activities that are used as needed. The first activity is an enhanced discussion of reasons to engage in ACP, with attention paid to developing the discrepancies between the most important reasons for engagement and lack of ACP participation. The second is addressing beliefs that serve as barriers to ACP. The third is an exercise to help patients clarify their values regarding quality versus quantity of life. Understanding how patients view this trade-off is central to decision making about potentially life-sustaining treatment ${ }^{22}$ and is included in a number of ACP tools ${ }^{23}$ as well as in the brochure provided with CTI. The fourth activity is a discussion of experiences that could help ACP engagement. If the participant has a surrogate who agrees to be part of the MI, the surrogate's perspectives are elicited in each of these steps. In pilot testing, sessions lasted a mean (SD) of 34.9 (6.5) minutes.

\section{Motivational enhancement therapy}

In the MET arm, participants are provided the CTI TTM-based feedback materials, and then engage in an MI interview as described previously, with the addition of review of the materials with the clinician. Each component of the interview makes mention as appropriate to specific sections of the stage-matched brochures to address knowledge gaps and provide more information about reasons to engage in ACP and addressing ACP barriers. In an additional section of the interview specific to MET, the clinician reviews the personalised feedback report with the participant to facilitate the development of a change plan.

\section{Control}

Participants in the control arm receive assessments at baseline, 2 months and 4 months but do not receive any additional information about ACP, other than that potentially provided in usual care. In order to minimise the effect of asking about ACP behaviours on participants' engagement in these behaviours, the control assessments conclude with questions about readiness to engage in and pros and cons of physical activity.

\section{Randomisation}

Randomisation in the community trial is at the level of the practice/senior living community in order to avoid contamination. Contamination could occur if patients assigned to the intervention increased their clinicians' awareness of ACP, and then clinicians changed 
their behaviours towards other participants potentially assigned to the control group. Therefore, randomisation occurs within matched pairs of sites, instead of at the individual level. Sites are matched according to available data regarding characteristics most likely to be associated with ACP participation. For clinical sites, this includes proportion of patients: age 55 years and older, non-white and with Medicaid. For senior living communities, this includes type of community (eg, continuing care retirement community) and, if applicable, type of contract (eg, life care and fee-for-service). After matching, sites are randomised by means of computer-based random number generator.

Because participants at the VA belong to a very large panel of providers, each of whom would have only a small number of patients in the study, randomisation is at the level of the patient. Number of ACP behaviours completed at baseline may be the single variable most highly associated with the likelihood of full ACP engagement, since engagement is cross-sectionally related to attitudes, beliefs and processes related to $\mathrm{ACP}^{11}$ and to a number of sociodemographic characteristics ${ }^{25}$ and life experiences. ${ }^{26}$ Therefore, in order to ensure balanced representation of number of ACP activities completed at baseline across the four study arms, participants are assigned using stratified permuted block randomisation with a block length of eight via a customised computer program that provides the assignment at the time of randomisation.

\section{Blinding}

Participants are not blinded to their assignment. Research assistants are not blinded to participant assignment at interim time points since the assessment, as described below, differs slightly according to the assignment. However, blinded research assistants ascertain the primary study outcome at the 6-month assessment.

\section{Measures}

The primary outcome is having completed, or being in the action/maintenance stage as specified in the TTM, for all of the four key ACP behaviours at 6 months. The designation of action and maintenance was originally designed for behaviours that required ongoing effort, such as smoking cessation and exercise, and refer to how long ago the behaviour was initiated. While there are activities that an individual can and should be doing during the maintenance phase of ACP, such as reviewing and updating documents, the focus for this study was on initial engagement in ACP. Therefore, these two stages were combined. The secondary outcomes are the stage of change for each of the behaviours. The stages of change prior to action/maintenance are: precontemplation, or not ready to take action within the next 6 months; contemplation, or thinking about taking action over the next 6 months; and preparation, or planning on taking action in the next 30 days. These variables are measured at each assessment: baseline, 2 months, 4 months and 6 months.
Sociodemographic, health and psychosocial status variables are assessed to describe the study population, test for the adequacy of randomisation and use as covariates. The sociodemographic status variables include: age, gender, race/ethnicity, level of education, income, marital status, housing type and living alone or with others. The health status variables include: self-rated health, ${ }^{27}$ self-rated quality of life and variables included in a validated prognostic index for 4-year mortality: current tobacco use, chronic conditions and functional status. ${ }^{28}$ The psychosocial status variables include: depression, measured using the Patient Health Questionnaire - 2 (PHQ-2), ${ }^{29}$ and religion, measured using the Duke University Religion Index ${ }^{30}$ and experience with surrogate medical decision making. ${ }^{26}$ These descriptive variables are measured at baseline only.

Additional constructs of the TTM are being assessed both as input for the expert system and as variables to be used in TTM-based models of ACP as behaviour change. These constructs are posited to be mediation variables in the pathway of behaviour change and include decisional balance, values/beliefs, confidence and processes of change. ${ }^{11}$ ACP knowledge is also assessed as a potential mediator. ${ }^{25}$ These variables are measured at each assessment.

The final 6-month assessment includes additional evaluation measures, asking participants how much participation in the study: increased their own interest in ACP, the interest of a significant other in ACP and was responsible for their own and/or a significant other's movement forward with ACP.

In order to minimise missing data, a shortened form of the 6-month assessment is available to those participants who do not want to complete a full assessment but are willing to answer an abbreviated set of items. This outcome assessment consists only of stage of change for the four ACP behaviours.

Participants recruited from community settings complete the baseline assessment as administered by a research assistant in person, unless they cannot stay after the appointment, in which case the assessment is completed by phone. They have the choice to complete follow-up assessments either by telephone or through self-administration. Both participants and research assistants access the assessment developed through the customisation of TTMX, proprietary behaviour change software licenced through Pro-Change Behavior Systems, Inc. The program is designed to support high-quality data collection by having respondents click on radio buttons next to responses to minimise data entry errors and not allowing respondents to leave a page until all questions are answered. Assessments can be completed up to 7 days prior to or 30 days following the target date. Participants recruited from the VA complete all assessments by telephone.

\section{Analytic plan}

The analysis for the community study is based on the study design of two groups (intervention and control) 
assessed on four occasions (baseline, 2, 4 and 6 months) with sites nested in groups based on cluster randomisation of matched pairs of sites. Baseline analyses will include examination of group differences to evaluate the success of the matched-pairs randomisation procedure and examination of potential covariates to reduce the expected within-groups dependency resulting from cluster randomisation.

The primary analysis will address the hypothesis that the proportion of participants in action/maintenance for the four ACP behaviours will be higher in the intervention group than in the control group at the 6-month assessment. Several analytical approaches are available within a more general framework of random effects modelling incorporating both time and site level effects in addition to potentially important covariates. The basic analytical approach will employ the generalised estimating equation (GEE) method to analyse intervention main effects and interaction (additive) effects. ${ }^{31}$ Analytic models will include interaction terms for time point and site. This will permit an examination of the effect of the intervention at the primary endpoint of 6 months and at the intermediate time points of 2 and 4 months.

The analysis for the VA study is based on the study design of four groups assessed on four occasions. The primary analysis for this study will address the hypothesis that the proportions of participants in action/maintenance for the four ACP behaviours will be higher in each intervention group than in the control group at the 6-month assessment. A logistic model that contains intervention group (CTI, MET, CTI+MET, with reference=control) as a categorical predictor will be used to analyse this outcome. The model will also control for the stratification variable (number of ACP activities completed at baseline) and for any factors found to be unbalanced across groups. Prespecified subgroup analyses will be conducted by using the same logistic modelling approach within each of the strata. Although the study is not powered to find significant differences between intervention arms or within strata, the study will provide preliminary data for future studies regarding the effect sizes for each intervention arm and potential differences in effectiveness within subgroups.

Analytic approaches for secondary outcomes will follow the same plan in both studies and be similar to those employed for the primary outcome variable. Both categorical and continuous secondary outcomes will be examined, including specific behavioural and intermediate outcome measures, such as the number of ACP behaviours changed and ACP attitudes. For continuous measures, multivariate analysis of variance, structural equation modelling, latent growth curve modelling and GEE techniques will be used. For categorical measures, latent transition analysis, logistic regression and GEE techniques will be used. For skewed frequency/count data, appropriate data transformation or Poisson regression approaches can be employed. Secondary analyses will also examine the nature of behaviour change within and across groups over time within the framework of multilevel structural equation modelling, including latent growth curve modelling, mediation modelling, cross-lagged panel designs and model invariance testing. ${ }^{32-36}$ These analyses will examine the relationships between intervention, mediator and moderator process measures. These analyses will provide considerable insight into how the interventions may be effecting change and will be especially helpful for continued development and refinement of the intervention. Mediation analysis in the control group will focus on how TTM constructs predict longitudinal adoption of ACP. Mediation analysis in the intervention group(s) will focus on how the TTM constructs and other sociodemographic factors compare as mediators of effective interventions. Because these modelling approaches can be complex, they will proceed in stepwise fashion systematically adding constructs and time points to more basic models to ensure model convergence. Models are built from the simplest to the most complex that the data and theory can support, using robust estimation methods and bias-corrected bootstrapping strategies for final model estimation and hypothesis testing.

\section{Sample size}

The sample size calculation for the community-based study was complicated by the use of practice site as the unit of treatment assignment and analysis, which introduces an unknown degree of dependency into the data. This dependency, or intraclass correlation (ICC), was conservatively assumed to be ICC $=0.05$ based on existing cross-sectional data, ${ }^{25}$ without covariate adjustment.

This cross-sectional data also provided an estimate of the prevalence of the primary outcome of between $4 \%$ and $8 \% .{ }^{25}$ We conservatively estimated that the prevalence of this outcome in the control group which, because of our exclusion criteria, will be $0 \%$ at baseline, will be $5 \%$ at the 6 -month assessment. The sample size is based on the ability to detect an absolute increase of $10 \%$ for the primary outcome in the treatment group over the control group, consistent with effect sizes in previous TTM-tailored interventions and a judgement regarding a minimum clinically significant effect size. Sample size calculations assumed one-tailed significance testing at alpha $=0.05$ and were based on a one-way analysis of variance for proportions with arcsine transformation and nested random effects for sites to accommodate the cluster randomised design. ${ }^{37}$ Based on an enrolment of a minimum of 16 sites for the study (eight matched pairs), to achieve power of 0.80 for the primary outcome, a final sample size of 50 individuals per site is needed, resulting in a final study sample size of 800 . Assuming $20 \%$ loss to follow-up, we estimate that a baseline sample size of 1000 for the community sample is required.

In the VA study, using the same estimates of $5 \%$ prevalence of the primary outcome in the control group, a sample size of 110 per group is required to detect an absolute increase of $10 \%$ for the primary outcome in each of the intervention groups to achieve a power of 0.80 with an 
alpha $=0.05$. Based on prior experience within VA samples, a lower $10 \%$ loss to follow-up was assumed, resulting in a baseline sample size of 121 per group, for a total of 484 .

\section{Patient and public involvement}

The ACP behaviours that are both the targets of intervention and the outcome measures for the STAMP studies were developed based on the input of older persons, their surrogate decision makers and bereaved caregivers obtained during focus groups ${ }^{10}$ and individual interviews. ${ }^{38}$ The assessment and printed intervention materials were pilot tested and modified in response to participant feedback, both about the content of the feedback and also regarding the length and burden of the assessment items. ${ }^{12}$ All procedures being used in the protocols were pilot tested in two practices, and modifications were made in response to feedback both from patients and physicians. There are no plans to disseminate the study results to participants.

\section{ETHICS AND DISSEMINATION}

\section{Ethics}

The study is being monitored with the use of a Safety Monitoring Committee. Members of this committee, with expertise in clinical geriatrics and study conduct, have reviewed and approved all study protocols and materials. Quarterly meetings occur to review any adverse events.

\section{Dissemination}

The results of the study will be presented to academic audiences through presentations at national meetings and publication in peer-reviewed journals. The principal investigator has partnered with leadership in both the community and VA settings, with ongoing discussion of how the STAMP interventions can be implemented, if shown to be efficacious.

\section{Author affiliations}

${ }^{1}$ Department of Medicine, Yale School of Medicine, New Haven, Connecticut, USA ${ }^{2}$ Clinical Epidemiology Research Center, VA Connecticut Healthcare System, West Haven, Connecticut, USA

${ }^{3}$ Cancer Prevention Research Center, College of Health Sciences, University of Rhode Island, Kingston, Rhode Island, USA

${ }^{4}$ Psychology Department, College of Health Sciences, University of Rhode Island, Kingston, Rhode Island, USA

${ }^{5}$ Department of Psychiatry, Yale School of Medicine, New Haven, Connecticut, USA ${ }^{6}$ Psychology Service, VA Connecticut Healthcare System, West Haven, Connecticut, USA

${ }^{7}$ Program on Aging, Yale School of Medicine, New Haven, Connecticut, USA

Contributors Study design: TRF, SM, CAR, AP and JSR; study conduct: TRF, SM, $\mathrm{LI}, \mathrm{MZ}$, LAB and J0; development of interventions: TRF, SM, CAR, AP, LI, MZ, LAB and J0; drafting of manuscript: TRF; review of manuscript for critical revisions: all authors; approval of final manuscript: all authors.

Funding This work is supported by the National Institute of Nursing Research (R01 NR016007), VA Health Services and Research (101 HX002062), National Institute of Aging (P30 AG21342) and National Center for Research Resources (G20 RR030883)

Disclaimer The funders have no role in the study design, collection, management, analysis and interpretation of data; writing of the report, or the decision to submit the report for publication. The views expressed in this article are those of the authors and do not necessarily represent the views of the Department of Veterans Affairs.

Competing interests None declared.

Patient consent Not required.

Ethics approval The protocol was reviewed and approved by the Institutional Review Boards of Bridgeport Hospital, which has governance over the majority of the community clinical sites participating in the study, and the Yale School of Medicine, which has governance over the remaining community sites, and the Human Subjects Subcommittee of VA Connecticut Healthcare System.

Provenance and peer review Not commissioned; peer reviewed for ethical and funding approval prior to submission.

Open access This is an open access article distributed in accordance with the Creative Commons Attribution Non Commercial (CC BY-NC 4.0) license, which permits others to distribute, remix, adapt, build upon this work non-commercially, and license their derivative works on different terms, provided the original work is properly cited, appropriate credit is given, any changes made indicated, and the use is non-commercial. See: http://creativecommons.org/licenses/by-nc/4.0/.

\section{REFERENCES}

1. Institute of Medicine. Dying in America: Improving and honoring individual preferences near the end of life. Washington, DC: The National Academies Press, 2014.

2. Chiarchiaro J, Buddadhumaruk P, Arnold RM, et al. Prior advance care planning is associated with less decisional conflict among surrogates for critically ill patients. Ann Am Thorac Soc 2015;12:1528-33.

3. Wright AA, Zhang B, Ray A, et al. Associations between end-of-life discussions, patient mental health, medical care near death, and caregiver bereavement adjustment. JAMA 2008;300:1665-73.

4. Yadav KN, Gabler NB, Cooney E, et al. Approximately one in three us adults completes any type of advance directive for end-of-life care. Health Aff 2017;36:1244-51.

5. Sudore RL, Fried TR. Redefining the "planning" in advance care planning: preparing for end-of-life decision making. Ann Intern Med 2010;153:256-61.

6. Detering KM, Hancock AD, Reade MC, et al. The impact of advance care planning on end of life care in elderly patients: randomised controlled trial. BMJ 2010;340:c1345

7. Kirchhoff KT, Hammes BJ, Kehl KA, et al. Effect of a disease-specific planning intervention on surrogate understanding of patient goals for future medical treatment. J Am Geriatr Soc 2010;58:1233-40.

8. Sudore RL, Boscardin J, Feuz MA, et al. Effect of the PREPARE website vs an easy-to-read advance directive on advance care planning documentation and engagement among veterans: a randomized clinical trial. JAMA Intern Med 2017;177:1102-9.

9. Wilson KS, Kottke TE, Schettle S. Honoring Choices Minnesota: preliminary data from a community-wide advance care planning model. J Am Geriatr Soc 2014;62:2420-5.

10. Fried TR, Bullock K, lannone L, et al. Understanding advance care planning as a process of health behavior change. J Am Geriatr Soc 2009;57:1547-55.

11. Fried TR, Redding CA, Robbins ML, et al. Promoting advance care planning as health behavior change: development of scales to assess decisional balance, medical and religious beliefs, and processes of change. Patient Educ Couns 2012;86:25-32.

12. Fried TR, Redding CA, Robbins ML, et al. Development of personalized health messages to promote engagement in advance care planning. J Am Geriatr Soc 2016;64:359-64.

13. Katzman R, Brown T, Fuld P, et al. Validation of a short orientationmemory-concentration test of cognitive impairment. Am J Psychiatry 1983;140:734-9.

14. Farmer CM, Hosek SD, Adamson DM. Balancing Demand and Supply for Veterans' Health Care: A Summary of Three RAND Assessments Conducted Under the Veterans Choice Act. Rand Health Q 2016;6:12.

15. U.S Department of Veterans Affairs. VA Telehealth Services. https:// www.telehealth.va.gov (accessed 20 Apr 2018).

16. Baban A, Craciun C. Changing health-risk behaviors: A review of theory and evidence-based interventions in health psychology. $J$ Consult Clin Psychol 2007;7.

17. Fried TR, Leung SL, Blakley LA, et al. Development and pilot testing of a motivational interview for engagement in advance care planning. J Palliat Med 2018;21:897-8. 
18. Ball SA, Martino S, Nich C, et al. Site matters: multisite randomized trial of motivational enhancement therapy in community drug abuse clinics. J Consult Clin Psychol 2007;75:556-67.

19. Carroll KM, Ball SA, Nich C, et al. Motivational interviewing to improve treatment engagement and outcome in individuals seeking treatment for substance abuse: a multisite effectiveness study. Drug Alcohol Depend 2006;81:301-12.

20. Martino S, Paris M, Añez L, et al. The effectiveness and cost of clinical supervision for motivational interviewing: a randomized controlled trial. J Subst Abuse Treat 2016;68:11-23.

21. Ball S, Martino S, Corvino J, et al. Independent Tape Rater Guide. Unpublished psychotherapy tape rating manual 2002

22. K. Heyland D, Pichora, D. The development and validation of a questionnaire to audit advance care planning. J Palliat Care Med 2012;02:119.

23. Green MJ, Levi BH. Development of an interactive computer program for advance care planning. Health Expect 2009;12:60-9.

24. Sudore RL, Knight SJ, McMahan RD, et al. A novel website to prepare diverse older adults for decision making and advance care planning: a pilot study. J Pain Symptom Manage 2014;47:674-86.

25. Fried TR, Redding CA, Robbins ML, et al. Stages of change for the component behaviors of advance care planning. J Am Geriatr Soc 2010;58:2329-36.

26. Amjad H, Towle V, Fried T. Association of experience with illness and end-of-life care with advance care planning in older adults. J Am Geriatr Soc 2014;62:1304-9.

27. Idler EL, Benyamini Y. Self-rated health and mortality: a review of twenty-seven community studies. J Health Soc Behav 1997;38:21-37.
28. Lee SJ, Lindquist $\mathrm{K}$, Segal MR, et al. Development and validation of a prognostic index for 4-year mortality in older adults. JAMA 2006;295:801-8.

29. Kroenke K, Spitzer RL, Williams JB. The Patient Health Questionnaire-2: validity of a two-item depression screener. Med Care 2003;41:1284-92.

30. Koenig HG, Büssing A. The Duke University Religion Index (DUREL): a five-item measure for use in epidemological studies. Religions 2010;1:78-85.

31. Zeger SL, Liang KY. Longitudinal data analysis for discrete and continuous outcomes. Biometrics 1986;42:121-30.

32. Fairchild AJ, MacKinnon DP. A general model for testing mediation and moderation effects. Prev Sci 2009;10:87-99.

33. Kaplan D. Structural equation modeling: foundations and extensions. 2nd ed. Thousand Oaks, CA: Sage, 2009.

34. Kline RB. Principles and practice of structural equation modeling. 4th ed. New York: Guilford Press, 2016

35. MacKinnon DP, Fairchild AJ, Fritz MS. Mediation analysis. Annu Rev Psychol 2007;58:593-614.

36. Rabe-Hesketh S, Skrondal A, Zheng X. Multilevel structural equation modeling. Lee S-Y, ed. Handbook of Computing and Statistics with Applications. Amsterdam: Elsevier, 2007.

37. Rossi JS, analysis S. Statistical power analysis. In: Schinka JA Velicer WF, eds. Handbook of psychology volume 2: research methods in psychology. 2nd edn. New York: John Wiley \& Sons, 2013;2:71-108.

38. Fried TR, O'Leary JR. Using the experiences of bereaved caregivers to inform patient- and caregiver-centered advance care planning. Gen Intern Med 2008;23:1602-7. 